\title{
Has the Antiplatelet Therapy a Role in the Prevention of Sudden Cardiac Death?
}

\author{
Juan C Garcia-Rubira*, and Manuel Almendro-Delia
}

Unidad de Gestion de Cardiologia, Hospital Universitario Virgen Macarena, Sevilla, Spain

\begin{abstract}
Coronary artery disease is a well-known cause of sudden cardiac death, and the key mechanisms are platelet aggregation and thrombosis. Furthermore, experimental studies have revealed that platelets have a direct influence on arrhythmic events. However, current antiplatelet therapy have failed to prevent sudden cardiac death in primary prevention trials, although a protective effect has been found in patients with advanced cardiovascular disease, reducing cardiac sudden death in this scenario.
\end{abstract}

Keywords: Aspirin; Antiplatelet therapy; Prevention; Sudden cardiac death

\section{Introduction}

It is widely recognized that coronary artery disease is the cause of a large proportion of cases of sudden cardiac death. There are several mechanisms involved in this relationship, which are summarized in a scheme proposed years ago by Robert Myerburg [1]. Firstly, a previously stable and silent atherosclerotic plaque may become unstable and cause transient ischemia. Secondly, an episode of plaque instability may be severe enough to cause an acute coronary syndrome, and finally an acute myocardial infarction. Third, the chronic phase is characterized by myocardial formation or scar. And finally, the infarcted left ventricle may suffer a remodelling process, which leads to ventricular dilatation and ventricular dysfunction. These four scenarios are subjected to the presence of environment modifiers: electrolytes, hemodynamic, neurohormonal imbalance and genetic predisposition. Any of these scenarios may be responsible for the episode of sudden cardiac death, either arrhythmic or mechanic (Figure 1).

The onset of these situations is platelet aggregation and thrombosis. Although platelet is the centre of thrombosis, its role in sudden cardiac death goes far beyond thrombosis [1,2]. Platelets contain three types of granules: dense granules (also called delta), alpha granules and lysosomes. When the platelets are activated, the granules fuse with the platelet membrane, releasing their contents and modifying the shape and functions of the cell. Delta granules have a high content of histamine, serotonin, adenosine diphosphate (ADP), adenosine triphosphate (ATP), guanosine triphosphate (GTP), guanosine diphosphate (GDP), calcium, and magnesium. Alpha granules contain more than 2000 different molecules, including P-selectin or the platelet factor 4 . In addition, they contain several receptors that are crucial in the process of thrombosis, as the glycoprotein $\alpha \operatorname{IIb} \beta 3$ receptor, which are exteriorized and activated with this process. Finally, following a more powerful stimulus, lysosomes content is released, namely modulator factors as proteases, cathepsin or glucohydrolases. These groups of molecules released from platelets are called "secretome". They have actions that can be involved in the production of arrhythmias, by a mechanism different from thrombosis (Figure 2,3,4).

In a recent study, Dhanjal et al. [3] investigated and proved this double role of platelets in the production of arrhythmias. They took a group of Wistar rats, from which they obtained blood and platelets. A portion of the platelet samples were stimulated to provoke the release of the secretome. Then they applied a model of ischemia producing a limited necrosis, in which only $30 \%$ of them had ventricular fibrillation, according to their previous experience. To produce entrapment of platelets in the infarct zone, they infused platelets directly in the coronary artery distal to the obstruction. They compared the effect of infused platelets with microspheres of the same size, or the infusion of the secretome previously obtained. Control animals had ventricular fibrillation in $30 \%$, as expected, while it occurred in $90 \%$ with platelet infusion. This result was not obtained neither with spheres of the same size nor with isolated secretome. As all the groups had arterial obstruction, a direct role of platelets beyond the obstruction is demonstrated. In a second part of the experiment, some of the animals received previous treatment with clopidogrel or indomethacin. When the blood was infused into the occluded artery there was a significant increase in ventricular fibrillation. This effect could not be prevented significantly with the previous treatment.

This study shows that we still have much to learn from platelets and their role in sudden death. But we already have a lot of clinical experience in the management of antiplatelet, and we need today clear instructions. In this sense we have to look at the clinical guidelines from the scientific societies. First, the European Guidelines on Cardiovascular Disease Prevention establish about preventive antiplatelet therapy [4]: "In the chronic phase (>12 months) after myocardial infarction, aspirin is recommended for secondary prevention" (Class I Recommendation). And "Aspirin or clopidogrel cannot be recommended in individuals without cardiovascular or cerebrovascular disease due to the increased risk of major bleeding" (Class III recommendation).

These recommendations are based on two publications that are pivotal: First, the Collaborative Meta-analysis of 2002 concerning secondary prevention [5]. In it the investigators included 287 studies with 212000 high-risk patients, comparing antiplatelet agents against placebo, or different antiplatelet regimens. Serious vascular events occurred in $10.7 \%$ of treated patients compared with $13.2 \%$ of controls.

*Corresponding author: Garcia-Rubira, Unidad Coronaria, Hospital Universitario Virgen Macarena, Avenida Dr Fedriani, Sevilla, Spain, Tel: 34 954008000; E-mail grubira1@gmail.com

Received October 24, 2014; Accepted December 24, 2014; Published January 01, 2015

Citation: Garcia-Rubira JC, Almendro-Delia M (2015) Has the Antiplatelet Therapy a Role in the Prevention of Sudden Cardiac Death?. Cardiol Pharmacol 4: 125. doi:10.4172/2329-6607.1000125

Copyright: (C) 2015 Garcia-Rubira JC. This is an open-access article distributed under the terms of the Creative Commons Attribution License, which permits unrestricted use, distribution, and reproduction in any medium, provided the original author and source are credited. 
Citation: Garcia-Rubira JC, Almendro-Delia M (2015) Has the Antiplatelet Therapy a Role in the Prevention of Sudden Cardiac Death?. Cardiol Pharmacol 4: 125. doi:10.4172/2329-6607.1000125

The authors conclude that "Aspirin or another antiplatelet drug is protective in patients with increased risk of occlusive vascular events, including: myocardial infarction, ischemic stroke, stable or unstable angina, old myocardial infarction, cerebral ischemia, peripheral vascular disease, or atrial fibrillation."

On the other hand, the negative recommendation regarding primary prevention is based on another meta-analysis from the same

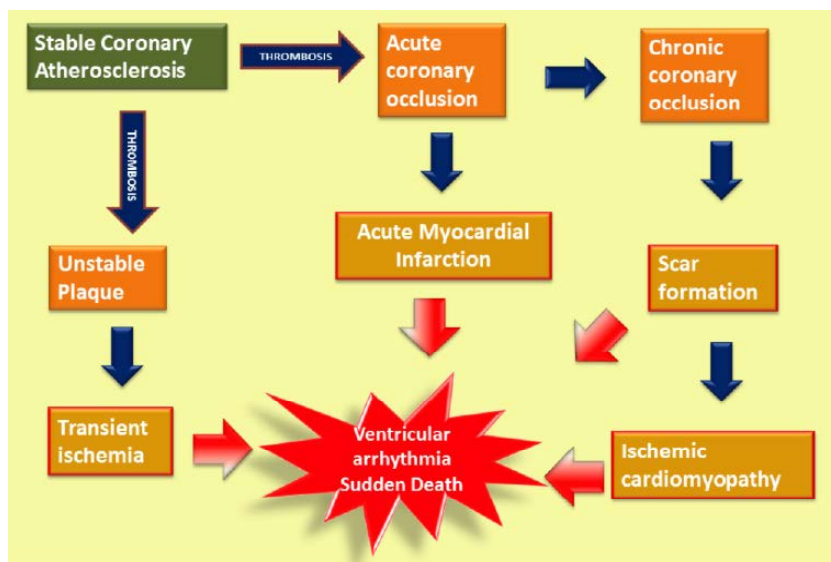

Figure 1: The four scenarios of coronary disease related to sudden cardiac death: transient ischemia, acute myocardial infarction, myocardial scar formation or scar and ventricular dysfunction. These four scenarios are subjected to the presence of environment modifiers [1].

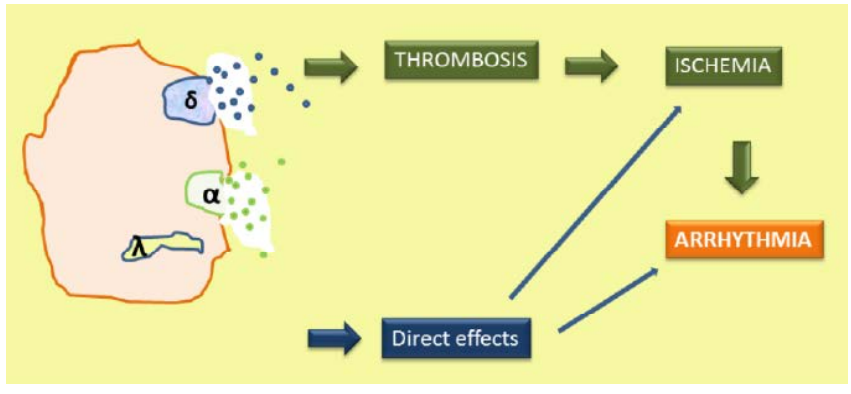

Figure 2: The double role of platelet on cardiac sudden death.

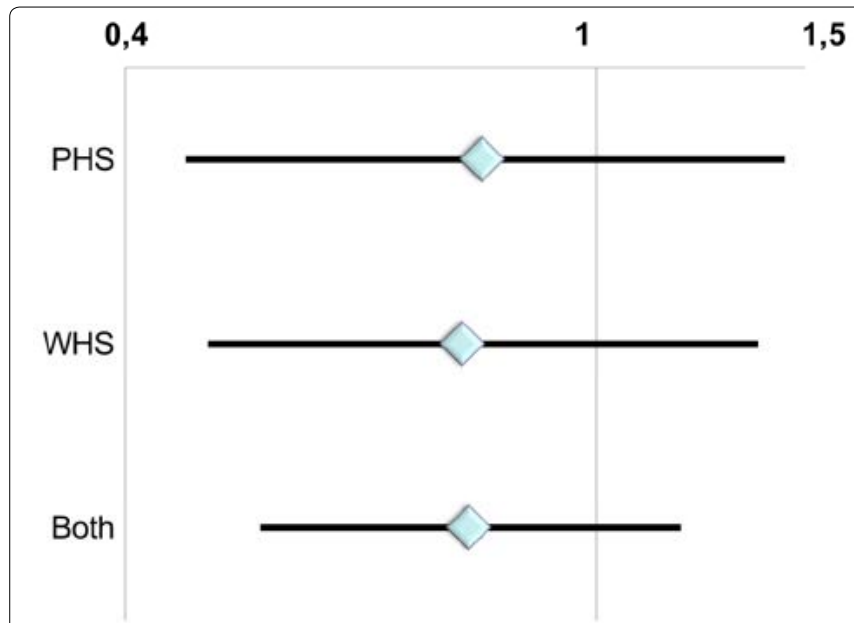

Figure 3: Effect of aspirin on sudden death according to the dataset from two primary prevention studies. Intervals show the hazard ratio. PHS: Physicians Health Study. WHS: Women's Health Study [11].

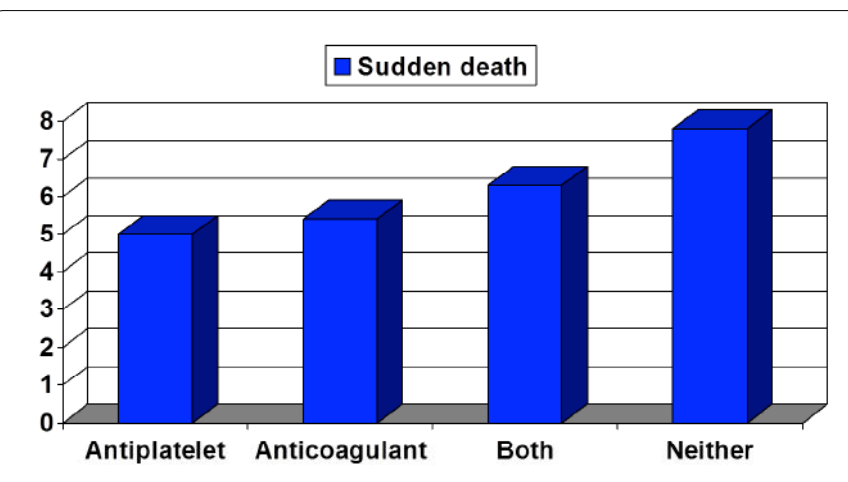

Figure 4: Effect of aspirine on sudden death in patients with left ventricular dysfunction from the SOLVD studies [12].

Colaborative Group, published in 2009 [6]. It included 95000 patients who had participated in 6 primary prevention trials, besides analysing separately several studies on secondary prevention. While the reduction of adverse cardiovascular events was significant, the magnitude was modest (from 0.57 to $0.51 \%$ per year). The cardiovascular mortality was not different, and the number of bleeding episodes was significantly higher.

However, this conclusion is not closed yet, and there are different recommendations in other scientific societies. Thus, the statement from the American College of Chest Physicians, also from 2012, states [7]: "For persons aged 50 years or older without symptomatic cardiovascular disease, we suggest low-dose aspirin 75 to $100 \mathrm{mg}$ daily over no aspirin therapy (Grade 2B recommendation)." This recommendation is based on a meta-analysis posterior to that from the Collaborative Group, and published in 2011. This study, from Raju et al. [8], included the previously mentioned 6 primary prevention trials, plus 3 other studies in which 7000 more patients were added. In total, they studied 100076 patients, finding for the first time a significant reduction in the mortality from all causes. As the favourable effect is seen almost exclusively in individuals older than 50 years, this condition is included in the recommendation.

But after analysing all these data, what can we say about the prevention of sudden death? Have any of the studies measured the sudden death as an outcome?

The truth is that most of the large trials concerning the preventive role of antiplatelets do not publish the incidence of sudden death in their cohorts. We have to go back to one of the first studies of primary prevention: The Physicians Health Study [9], which studied 22000 physicians aged 40-84, with a follow up of 60 months, and the Women Health Study [10], which studied 39800 women over 45 years, with a follow up of 10 years. The data concerning sudden death of both studies were presented at the American College Congress in 2013 [11]. Among the more than 60.000 participants followed for 5-10 years, 91 cases of sudden death were recorded. No significant difference was found between aspirin or placebo.

Concerning the issue of secondary prevention, we find again that it is not easy to find data concerning the effect of antiplatelet agents in sudden death. In the SOLVD studies, 6797 patients with left ventricular dysfunction were included with a mean follow up of 39.9 months [12]. At baseline, $46 \%$ had antiplatelet therapy, $13 \%$ anticoagulants, and $2 \%$ both. The antiplatelet therapy was associated with a significant reduction in total mortality, cardiovascular mortality, and sudden death. The reduction in sudden death remained significant 
Citation: Garcia-Rubira JC, Almendro-Delia M (2015) Has the Antiplatelet Therapy a Role in the Prevention of Sudden Cardiac Death?. Cardiol Pharmacol 4: 125. doi:10.4172/2329-6607.1000125

Page 3 of 3

in the multivariate analysis. Another study that offers some data is the Veterans Cooperative Study of diabetic patients with severe vascular disease [13]. It included 231 diabetic patients with gangrene or amputation due to vascular causes. Treatment with dipyridamole plus aspirin reduced significantly the incidence of sudden death. Although these data are suggestive, we do not know if these findings can be generalized to the population with cardiovascular disease. On the other hand, this population already has indication for antiplatelet therapy, making it impractical to do randomized studies versus placebo.

\section{Conclusion}

Experimental studies have clearly shown that platelets have a role on sudden cardiac death, not only through the thrombotic occlusion of coronary arteries, but also an arrhythmic role. However, current antiplatelet agents have not proven to be effective in the primary prevention of sudden cardiac death. Some studies suggest a role of them in secondary prevention.

\section{References}

1. Myerburg RJ, Junttila MJ (2012) Sudden cardiac death caused by coronary heart disease. Circulation 125: 1043-1052.

2. de Jong JSS, Dekker LRC (2010) Platelets and cardiac arrhythmia. Frontiers in Physiology 166: 1-6.

3. Dhanjal TS, Medina RA, Leem J, Clark JE, Southworth R, et al. (2013) Trapped platelets activated in ischemia initiate ventricular fibrillation. Circ Arrhythm Electrophysiol 6: 995-1001.

4. Perk J, De Backer G, Gohlke H, Graham I, Reiner Z, et al. (2012) European Association for Cardiovascular Prevention \& Rehabilitation (EACPR); ESC Committee for Practice Guidelines (CPG). European Guidelines on cardiovascular disease prevention in clinical practice (version 2012). The Fifth Joint Task Force of the European Society of Cardiology and Other Societies on Cardiovascular Disease Prevention in Clinical Practice (constituted by representatives of nine societies and by invited experts). Eur Heart J 33: 16351701.

5. Antithrombotic Trialists' Collaboration (2002) Collaborative meta-analysis of randomised trials of antiplatelet therapy for prevention of death, myocardial infarction, and stroke in high risk patients. BMJ 324: 71-86.

6. Antithrombotic Trialists' (ATT) Collaboration, Baigent C, Blackwell L, Collins R Emberson J, et al. (2009) Aspirin in the primary and secondary prevention of vascular disease: collaborative meta-analysis of individual participant data from randomised trials. Lancet 373: 1849-1860.

7. Vandvik PO, Lincoff AM, Gore JM, Gutterman DD, Sonnenberg FA, et al. (2012) Primary and secondary prevention of cardiovascular disease: Antithrombotic Therapy and Prevention of Thrombosis, 9th ed: American College of Chest Physicians Evidence-Based Clinical Practice Guidelines. Chest 141: e637S668 S.

8. Raju N, Sobieraj-Teague M, Hirsh J, O’Donnell M, Eikelboom J (2011) Effect of aspirin on mortality in the primary prevention of cardiovascular disease. Am J Med 124: 621-629.

9. Final report on the aspirin component of the ongoing Physicians' Health Study (1989) Steering Committee of the Physicians' Health Study Research Group. N Engl J Med 321: 129-135.

10. Ridker PM, Cook NR, Lee IM, Gordon D, Gaziano JM, et al. (2005) A randomized trial of low-dose aspirin in the primary prevention of cardiovascular disease in women. N Engl J Med 352: 1293-1304.

11. Tung P, Van Denburgh M, Buring J, Gaziano JM, Manson J, et al. (2013) Aspirin, Vitamin E and Risk of Sudden Cardiac Death in Healthy Individuals. J Am Coll Cardiol 61: E1339.

12. Dries DL, Domanski MJ, Waclawiw MA, Gersh BJ (1997) Effect of antithrombotic therapy on risk of sudden coronary death in patients with congestive heart failure. Am J Cardiol 79: 909-913.

13. Colwell JA, Bingham SF, Abraira C, Anderson JW, Comstock JP, et al (1989) V.A. Cooperative Study of antiplatelet agents in diabetic patients after amputation for gangrene: unobserved, sudden, and unexpected deaths. J Diabet Complications 3: 191-197. 